\title{
ON A QUARANTINE MODEL OF CORONAVIRUS INFECTION AND DATA ANALYSIS
}

\author{
Vitaly Volpert ${ }^{1,2,3, *}$, Malay BanerjeE ${ }^{4}$ and Sergei PetrovskiI ${ }^{5}$
}

\begin{abstract}
Attempts to curb the spread of coronavirus by introducing strict quarantine measures apparently have different effect in different countries: while the number of new cases has reportedly decreased in China and South Korea, it still exhibit significant growth in Italy and other countries across Europe. In this brief note, we endeavour to assess the efficiency of quarantine measures by means of mathematical modelling. Instead of the classical SIR model, we introduce a new model of infection progression under the assumption that all infected individual are isolated after the incubation period in such a way that they cannot infect other people. Disease progression in this model is determined by the basic reproduction number $\mathcal{R}_{0}$ (the number of newly infected individuals during the incubation period), which is different compared to that for the standard SIR model. If $\mathcal{R}_{0}>1$, then the number of latently infected individuals exponentially grows. However, if $\mathcal{R}_{0}<1$ (e.g. due to quarantine measures and contact restrictions imposed by public authorities), then the number of infected decays exponentially. We then consider the available data on the disease development in different countries to show that there are three possible patterns: growth dynamics, growth-decays dynamics, and patchy dynamics (growthdecay-growth). Analysis of the data in China and Korea shows that the peak of infection (maximum of daily cases) is reached about 10 days after the restricting measures are introduced. During this period of time, the growth rate of the total number of infected was gradually decreasing. However, the growth rate remains exponential in Italy. Arguably, it suggests that the introduced quarantine is not sufficient and stricter measures are needed.
\end{abstract}

Mathematics Subject Classification. 92D30.

Received March 16, 2020. Accepted March 16, 2020.

\section{SIR MODEL}

The classical susceptible-infected-recovered (SIR) model in epidemiology (see, e.g., [2]) allows the determination of critical condition of disease development in the population irrespective of total population size. Among

Keywords and phrases: Coronavirus infection, quarantine model, modes of infection development.

${ }^{1}$ Institut Camille Jordan, UMR 5208 CNRS, University Lyon 1, 69622 Villeurbanne, France.

2 INRIA Team Dracula, INRIA Lyon La Doua, 69603 Villeurbanne, France.

3 Peoples Friendship University of Russia (RUDN University), 6 Miklukho-Maklaya St, Moscow 117198, Russia.

4 Department of Mathematics \& Statistics, IIT Kanpur, Kanpur 208016, India.

5 School of Mathematics \& Actuarial Science, University of Leicester, Leicester LE1 7RH, UK.

* Corresponding author: volpert@math.univ-lyon1.fr 
many versions of the model, let us consider the following ODE system

$$
\begin{gathered}
\frac{\mathrm{d} S}{\mathrm{~d} t}=-k I S, \\
\frac{\mathrm{d} I}{\mathrm{~d} t}=k I S-\beta I-\sigma I, \\
\frac{\mathrm{d} R}{\mathrm{~d} t}=\beta I,
\end{gathered}
$$

for the sizes (numbers) of the susceptible sub-population $S$, infected $I$, and recovered $R$. The term $k I S$ describes the disease transmission rate due to the contact with infected, $\beta I$ characterizes the rate of recovery of infected, and $\sigma I$ mortality due to infection only. It is assumed here that recovered do not return to susceptible class, that is, recovered individuals have immunity against new infection, and they cannot infect susceptible.

Analysis of this model is well known and we will not discuss it here. Let us only determine the condition of the disease progression in the case where the number of infected/recovered/dead is much less than the number of susceptible, and $S$ in the model above can be approximate by a constant, $S \approx S_{0}$. The equation (1.2) becomes as follows:

$$
\frac{\mathrm{d} I}{\mathrm{~d} t}=k I S_{0}-\beta I-\sigma I
$$

We obtain an ordinary differential equation with constant coefficients whose solution can be readily found, $I(t)=I_{0} e^{\mu t}$, where $I_{0}$ is the number of infected at the initial moment of detection of the infection/disease. Substituting this expression into equation (1.4), we get

$$
\mu=\left(k S_{0}-\beta-\sigma\right)=(\beta+\sigma)\left(\mathcal{R}_{0}-1\right),
$$

where the new parameter $\mathcal{R}_{0}=k S_{0} /(\beta+\sigma)$ is called the basic reproduction number. If $\mathcal{R}_{0}>1$, then the number of infected will grow, if $\mathcal{R}_{0}<1$ it will decay which plays the key role behind disease propagation.

In particular, one can change the course of the disease dynamics by changing the basic reproduction number. For instance, if measures are introduced that push it below one (e.g. by making $k$ to decrease), the exponential growth changes to exponential decay. Interestingly, this is exactly what has happened in South Korea (see Sect. 3) after restrictions on daily life were introduced.

Thus, the classical SIR model determines the condition of the disease development from the comparison of the disease transmission rate with the sum of the recover and death rates. In the other words, we compare the rates of adding and removal of infected.

The model does not account for the incubation period of the disease that was shown to be important in the case of coronavirus spread: individuals can become infective before showing any symptoms. A model that allows for the incubation period is considered in the next section.

\section{QuARANTINE MODEL}

\subsection{Model}

Exceptional measures adopted for the coronavirus infection suggest to introduce another model of infection development. We consider the sub-population of latently infected individuals who are already infected but do not show any symptom during the incubation period. When the incubation period is over, the disease manifests 
itself with its symptoms, and the individual is isolated in the quarantine where he/she cannot infect the others. Under these assumptions, instead of system (1.1)-(1.3) we have

$$
\begin{gathered}
\frac{\mathrm{d} S}{\mathrm{~d} t}=-k I(t) S(t), \\
\frac{\mathrm{d} I}{\mathrm{~d} t}=k I(t) S(t)-k I(t-\tau) S(t-\tau),
\end{gathered}
$$

where $I$ is the sub-population of latently infected individuals, $\tau$ is the incubation period. The second term in the right-hand side of equation (2.2) corresponds to the individuals infected at time $t-\tau$. Their incubation period is finished at time $t$, and they are put to quarantine. As a result they cannot spread infection anymore.

\subsection{Solution}

As before, we approximate $S(t) \approx S_{0}, S(t-\tau) \approx S_{0}$ as $\tau$ is not large, and replace equation (2.2) by the equation

$$
\frac{\mathrm{d} I}{\mathrm{~d} t}=k I(t) S_{0}-k I(t-\tau) S_{0}
$$

Substituting $I(t)=I_{0} e^{\mu t}$ in above equation, we get

$$
\mu=k S_{0}\left(1-e^{-\mu \tau}\right) .
$$

This equation has solution $\mu=0$ for all values of parameters. Besides this solution, it can have a positive or a negative solution $\mu$. The function $f(\mu)=k S_{0}\left(1-e^{-\mu \tau}\right)$ is an increasing function with the asymptotic limit $k S_{0}$ at infinity. The sign of the solution is determined by the derivative $f^{\prime}(0)$. If $f^{\prime}(0)>1$, then there is a positive solution (Fig. 1), if $f^{\prime}(0)<1$, the solution is negative. In terms of the basic reproduction number $\mathcal{R}_{0}=k S_{0} \tau$, the condition is similar as for the SIR model. However, the meaning of this parameter is different. It characterizes the total infection rate during the incubation period and not its ratio with the rates of recover and death.

With the notation $\hat{\mu}=\mu /\left(k S_{0}\right)$, we can write equation (2.4) as follows:

$$
\hat{\mu}=1-e^{-\mathcal{R}_{0} \hat{\mu}}
$$

Its solution depends on the single parameter $\mathcal{R}_{0}$.

\subsection{Public health}

In the absence of vaccination and lack of effective treatment, the only way to influence the disease development is to act on the basic reproduction number $\mathcal{R}_{0}=k S_{0} \tau$, that is to decrease the value of the parameter $k$ characterizing the disease transmission rate by the infected individuals (among which the symptoms are not prominent yet). Note that $S_{0}$ is a characteristic of the given population and $\tau$ is the property of the disease, therefore neither of them is controllable. However, parameter $k$ depends on the human mobility and the social distance and hence it can be changed. If in the beginning of the disease development $k>1 /\left(S_{0} \tau\right)$, that is each infected individual infects on average more than one susceptible individual during the incubation period, then the number of newly infected individuals will grow exponentially. If, at some moment of time, there are measures restricting potential contacts of infected and susceptible individuals, then $k$ can become less than the critical value, and the number of newly infected individuals will exponentially decay. There is a transition between the growing and decaying exponential. 


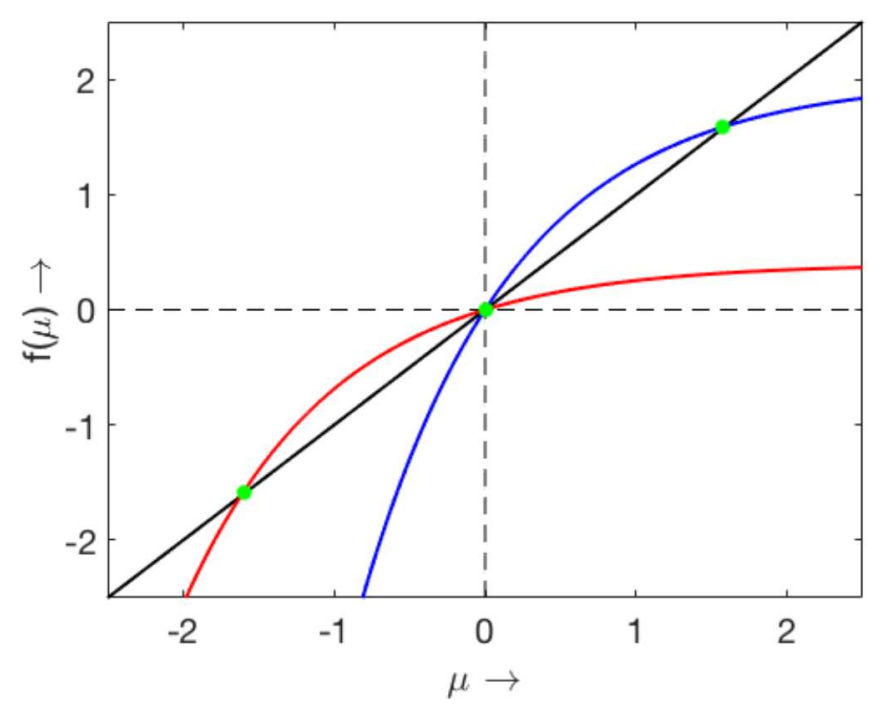

FIGURE 1. Graphical solution of equation $(2.4), \mu=f(\mu)$ with the values of parameters: $\tau=1$, $k S_{0}=2$ (dotted curve) and $k S_{0}=0.4$ (dashed curve).

\subsection{Limitations of the model}

The model has a number of obvious limitations. It takes into account only the beginning of the disease development where the number of suspected can be considered as constant. It is a good approximation if the disease propagation is stopped/regulated when the number of infected is relatively small (compared to the total population).

Next, the model does not take into account the spatial distribution of infected and their displacement. In this case, instead of equation (2.3) one has to consider a model with an explicit space, for instance the following equation:

$$
\frac{\partial I(x, t)}{\partial t}=\delta \frac{\partial^{2} I(x, t)}{\partial x^{2}}+k I(x, t) S_{0}-k I(x, t-\tau) S_{0}
$$

where $I$ and $S_{0}$ now have the meaning of the corresponding densities instead of sizes and the diffusion term describes random motion of the individuals with intensity $\delta$. In the case where neither of $S_{0}, k$ and $\tau$ depend on space, equation (2.6) can be reduced to the nonspatial model. If we consider, for simplicity, the whole axis (or a bounded interval with no-flux boundary conditions), then we introduce the variable $J(t)=\int_{-\infty}^{\infty} I(x, t) \mathrm{d} x$. Integrating equation (2.6) with respect to $x$, we obtain an equation similar to (2.3).

However, the spatially averaged model does not describe the dynamics well in case some of the parameters depend on space. If there are two different patches of the disease development with different basic reproduction numbers, then the disease can be eradicated in the first patch due to the imposed restrictions but it can give a new outbreak in another patch if the restrictions are not adopted there or they are not sufficient. This situation is observed in Europe where the disease progresses exponentially while it is already slowed down in China. Hence, in some cases, a multi-patch model should be considered with some connectivity between the patches at least for some time period. Also, the human movement can follow a pattern more complicated than the one given by the Fickian diffusion, e.g. to follow the network made by long-distance connections between large airports.

Another important limitation of the model is related to the assumption of a single incubation period. According to the data, it is possible that there are different incubation periods or maybe the whole spectrum of incubation periods from several days up to four weeks. The distributed delay models are appropriate in this case. 


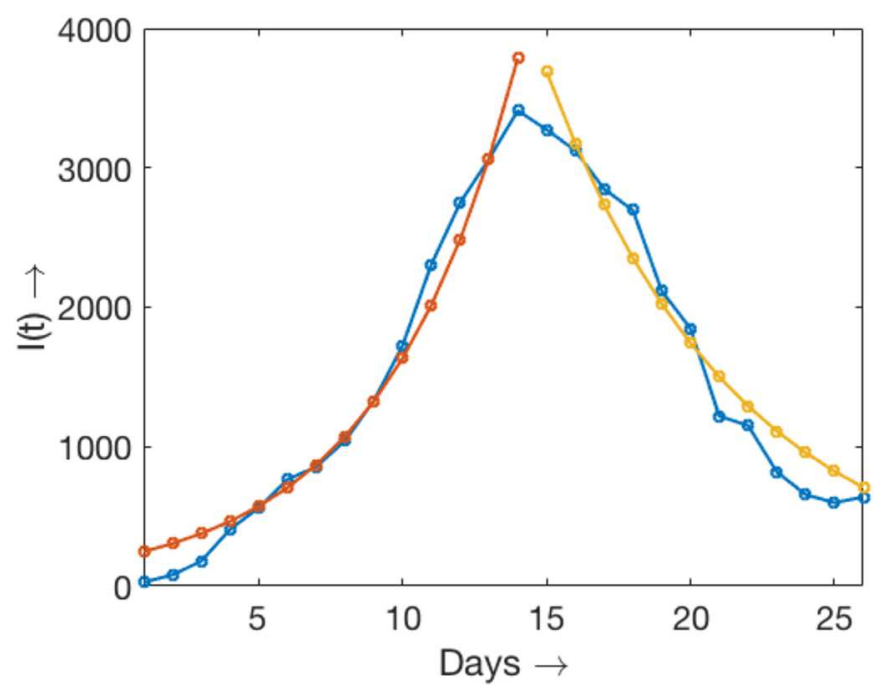

FIgURE 2. Latently infected individuals in South Korea obtained as a sum of daily cases during the incubation period taken equal 5 days. The growing branch is approximated by the exponential $200 e^{0.21 x}$, decaying exponential by $35 \cdot 10^{3} e^{-0.15 x}$.

Finally, let us mention virus mutations that can have a strong influence on the disease progression and treatment [1]. At the moment, there are no available data on mutations of coronavirus, it may take some time to have confirmation of this aspect.

\section{DATA}

We used the data from Worldometer ${ }^{1}$ showing the total number of infected individuals and daily cases in different countries and worldwide. Across the world daily cases clearly show two-patch dynamics basically due to China (growth-decay) and Europe (growth). There are periods of exponential growth, decay and re-growth. The origin of the outbreak on February 12 (in China) is not clear yet. It may be related to the method of data collection. Daily reported cases curves in China and South Korea correspond to the growth-decay dynamics. The daily new cases curves for France, Germany, Italy, Spain, USA correspond to the growth mode.

Let us note that the data on daily reported cases do not exactly correspond to the variable $I(t)$. The latter represents a sum of daily cases during the incubation period. Taking into consideration that the incubation period to be of 5 days, we obtain the graphs for the latently infected individuals. We observe growth-decay dynamics for South Korea (Fig. 2) and growth dynamics in Italy (Fig. 3).

Fitting the data allows us to determine the basic reproduction number from equation (2.5). For South Korea, $\mathcal{R}_{0}=1.123$ on the growing branch and $\mathcal{R}_{0}=0.932$ on the decaying branch. For Italy, $\mathcal{R}_{0}=1.103$. Let us note that the basic reproduction number depends on the incubation period.

\section{Discussion}

The model confirms the efficiency of the approach to stop the disease by the limitation of contacts between the individuals through quarantine of infected individuals. This is quite obvious in theory, with the help of simplest model formulation, but difficult in practice. Experience of China and South Korea shows that the peak of infection (maximum of newly reported cases on daily basis) is reached about 10 days after adopting serious restrictive measures. The number of infection increased during this time in 10-20 times. In Italy 10 days

\footnotetext{
Ihttps://www.worldometers.info/coronavirus/
} 


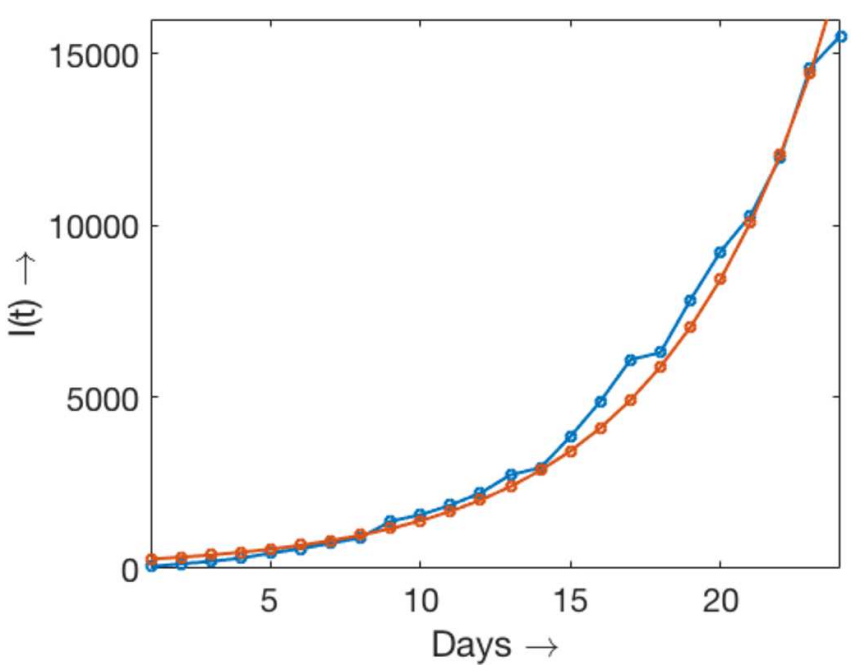

FIGURE 3. Latently infected individuals in Italy obtained as a sum of daily cases during the incubation period taken equal 5 days. The data curve is approximated by the exponential $230 e^{0.18 x}$.
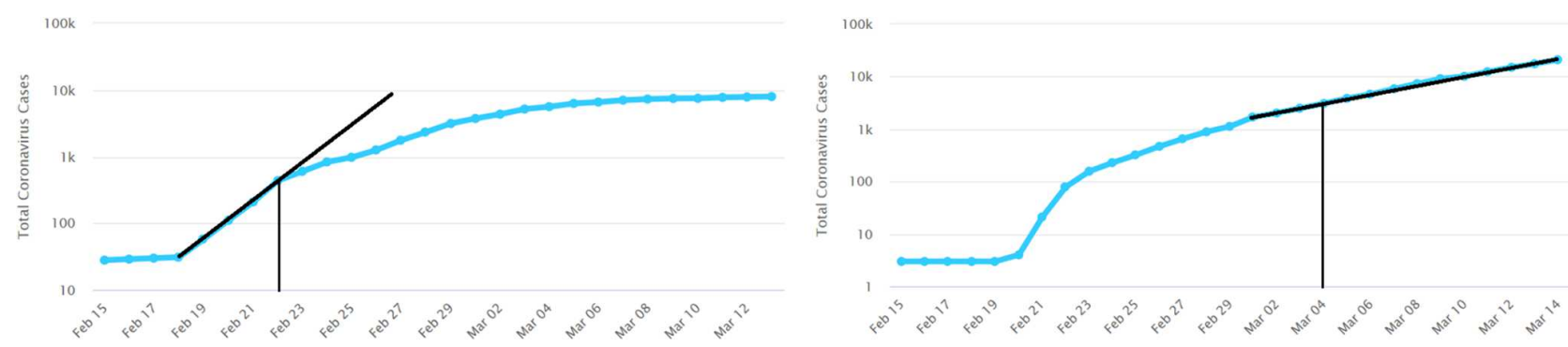

Figure 4. Total number of infected in the logarithmic scale. In South Korea (left), the exponential growth rate (linear growth in this scale) before February 22 is replaced by a slower growth after this date. In Italy (right), the exponential growth rate (linear growth in this scale) after March 4 does not change.

after the universities and schools were closed (March 4) the peak of infection does not seem to be reached, and exponential growth continues.

Moreover, the exponential growth rate of the total number of infected in China and in South Korea observed before the adopted measures (January 25 and February 22, respectively) rapidly changed to a slower growth rate afterward (Fig. 4). Similar situation is observed in Iran though the information about adopted measures is not clear yet. However, in Italy the exponential growth rate does not change up to March 4 . This can be an indication that these measures are not sufficient (or that they are not respected by local people).

Acknowledgements. The first author acknowledges the IHES visiting program during which this work was done. The work was supported by the Ministry of Science and Education of Russian Federation, project number FSSF-2020-0018, and by the French-Russian program PRC2307.

\section{REFERENCES}

[1] N. Bessonov, G. Bocharov, C.C. Leon, V. Popov and V. Volpert, Genotype dependent virus distribution and competition of virus strains. To appear in Memocs (2020).

[2] J. Murray, Vol.1 of Mathematical biology, third edition. Springer-Verlag, Heidelberg (2002). 